\title{
Genetic polymorphism of matrix metalloproteinases in breast cancer
}

\author{
Minireview
}

E. WIECZOREK, E. RESZKA, J. GROMADZINSKA, W. WASOWICZ*

Department of Toxicology and Carcinogenesis, Nofer Institute of Occupational Medicine, 91-348 Lodz, Poland

*Correspondence: wojciech@imp.lodz.pl

Received November 3, 2011 / Accepted December 19, 2011

\begin{abstract}
The family of human matrix metalloproteinases (MMPs) consists of 24 zinc- and calcium-dependent proteolytic enzymes. MMPs are divided into six subgroups, in terms of differences in the substrate specificity with structural domain architecture. These enzymes are involved in many physiological processes, such as skeletal development, wound healing, scar formation, as well as carcinogenesis. MMPs, fulfilling its function of degradation of extracellular matrix components, are involved in one of the stages of angiogenesis enabling the development, growth and spread of the primary tumor. Therefore, the search for the common polymorphic variants of MMPs, new genetic markers as prognostic factors in breast cancer progress seems to be understandable.

The minireview presents the results of 19 case-control or prospective studies concerning the association of SNPs of genes encoding nine MMPs: MMP-1, $-2,-3,-7,-8,-9,-12,-13,-21$ with the breast cancer risk, progression and survival.
\end{abstract}

Key words: matrix metalloproteinases, genetic polymorphism, breast cancer

Breast cancer, one of the leading civilization diseases, has for a number of years already focused attention of clinicians and researchers. It is the most commonly diagnosed cancer in women and is the major cause of death among female cancer patients. GLOBCAN estimates that, among the 12.7 million cancer cases worldwide in 2008, 2.9 million were women with breast cancer, and a million died of this cancer [1]. It is estimated that approximately $10 \%$ of the incidence of breast cancer is genetically-related and in most of the cases results from mutations in the genes BRCA1, BRCA2, $p 53$, ATM. Attention should also be paid to a number of environmental factors related to diet and occupational exposure, including tobacco smoking, alcohol intake, obesity, shift work at night. Factors considered in the etiology of breast cancer include also endogenous hormonal factors, such as age of occurrence of menarche, menopause, number of births, and exogenous hormonal factors such as use of hormonal contraceptives or hormonal replacement $[2,3]$. Currently, a description of cancer cases includes clinical stage and Tumor Nodus Metastases (TNM) clinical classification, hormone receptor status and
BRCA1, BRCA2 mutations. All these indicators are still not sufficient to predict the course of the cancer and, therefore, it is important to search for new markers that will enable the patient's prognosis and new predictive markers of cancer risk. As the majority of breast cancer deaths are caused by the invasion of cancer to other, sometimes distant organs, it is believed that it is appropriate to study the role of the enzyme gene polymorphisms that affect the process of angiogenesis largely responsible for the development, growth and metastatic capacity of the primary tumor. Key enzymes involved in these processes include the matrix metalloproteinases (MMPs) participating in an important stage of angiogenesis, namely the degradation of extracellular matrix (ECM) components.

Matrix metalloproteinases. MMPs are proteolytic enzymes whose enzymatic activity depends on zinc and calcium ions. MMPs were discovered in 1962 by Gross and Lapiere, who studied collagenolytic activity in amphibian tissues [4]. MMPs comprise a large family of extracellular proteinases. So far, 24 types of proteolytic enzymes have been found in humans [5]. 
The basic structural characteristics of these enzymes include a conservative amino acids featuring motif HEXXHXXGXXH (where $\mathrm{H}$ is histidine, E-glutamic acid, G-glycine, $\mathrm{X}$-any amino acid). This catalytic domain binding the zinc ions forms a unique "Met-turn" structure, responsible for the stability and enzymatic activity. Differences in the structural architecture and substrate specificity of MMPs have made it possible to classify MMPs into six subgroups. These subgroups include collagenases, gelatinases, stromelysins, matrilysins, membrane-type MMPs (MT-MMPs) and other MMPs [6]. Substrates for MMPs include both matrix substrates i.e. different types of collagen, gelatin, fibronectin, vitronectin, laminin, and also bioactive substrates, such as interleukin $1 \beta$ and proMMP-9 [7].

Synthesis of MMPs depends on various factors that may either activate or inhibit the process. MMPs are initially synthesized in cells in the form of preproenzymes and are released as zymogens (proMMPs) into the ECM, except MT-MMPs. Under normal conditions, proMMPs are synthesized by fibroblasts, leukocytes, monocytes, macrophages, neutrophils, endothelial cells and keratinocytes, and in pathological conditions by tumor cells. It has been shown that molecules on the surface of cancer cells stimulate nearby fibroblasts to produce MMP-1, MMP-2, MMP-3, and proMMP-2 activators [8]. Proteolytic activation of proMMPs involves unfolding the active site, as a result of isolating the prodomain from the catalytic domain. Activation and inhibition of MMPs may occur in several stages, ranging from induction of gene transcription, through proenzyme activation to inhibition of active MMPs [9].

Role of matrix metalloproteinases. MMPs are essential in physiological processes, such as normal development of the skeleton, supporting tissues, repair, wound healing, scar formation, and also participate in the pathogenesis of osteoporosis and the process of carcinogenesis. Their role in disease progression consists primarily in the degradation of the ECM. MMPs contribute to tumor angiogenesis that form new blood vessels, cell adhesion and epithelial to mesenchymal transition. MMPs catalyze the hydrolysis a variety of ECM macromolecules, and structural component of blood vessel basement membranes, destroy the structural barrier preventing the migration of cancer cells and cells involved in the process of morphogenesis. Proteolysis of structures located in the ECM and the breakdown of blood vessel basement membranes allows primary tumor growth and formation of metastatic sites. In addition to degradation of structural components of the microenvironment, MMPs can release from the ECM the signaling molecules, such as cytokines, growth factors and their receptors so important in tumor growth and angiogenesis. These compounds may also be proteolytically converted by MMPs $[10,11,12]$. It has been also found that MMPs inhibit immune responses against tumor cells by damaging interleukin 2 receptors on T cells [13].

Single nucleotide polymorphisms. Changes in DNA sequence occurring between individuals of the species and found in more than $1 \%$ of people in the population, are called single nucleotide polymorphisms (SNPs). So far, ten million SNPs that occur on average once every few hundred base pairs have been identified in the human genome $[14,15]$. Location of SNPs may affect the efficiency of the process of gene transcription, mRNA stability and may lead to amino acid substitution in the encoded protein, which in turn can alter the function of encoded proteins or their expression at the level of gene and protein.

Identification of proper common genetic variants is a complex and difficult task, which involves the fields of epidemiology and molecular biology. The study of SNPs, among other problems, seeks to identify genetic markers reflecting the risk of the disease. It is believed that the vast majority of SNPs are functionally neutral, and only their small proportion is functionally essential and may contribute to such interindividual variability in the susceptibility to developing various diseases, including cancer. Determination of polymorphisms that predispose to the development of cancer involves examination of the frequency of genotypes in a large and homogeneous population [16]. It is also believed that common genetic variants should also be considered when assessing the prognosis and the treatment of cancer. Such analysis can help to improve the efficiency of detection of cancer, or identify high-risk groups by the use of polygenic tests during implementation of a screening program. The analyses performed currently in the patients to determine the histological type of tumor, hormone receptor status and mutations, are not able to provide reliable prognosis for the effects of applied therapy or disease-free survival [17]. Recent studies on the functional importance of SNPs in the promoter sequence of genes encoding various MMPs have shown their significance for the risk of developing and persistence of many lifestyle-related common diseases, such as coronary heart disease, aneurysms, arthritis, periodontal disease or cancer $[18,19]$.

Characteristics of selected MMPs in breast cancer. The role of MMPs in breast cancer has not been clearly explained, although it has been analyzed by many authors [20, 21]. Researchers who examine the significance of MMPs polymorphisms have attempted planned selection of individual SNPs. While performing that MMPs targeted genotyping, the authors took into account primarily proven mechanisms describing the impact of functional polymorphisms on the MMPs gene transcriptional activity (Table 1). The selected SNPs were also previously analyzed in other cancers, such as lung, stomach, ovarian and bladder cancer. The results of these studies point to a positive correlation between MMPs polymorphism and the susceptibility to develop cancer, tumor invasiveness and further progression of the disease $[22,23]$.

Studies on the association between genetic polymorphism of MMPs and the risk of breast cancer, progression and diseasefree survival that have been performed so far are concerned with the following MMPs: MMP-1, MMP-8 and MMP-13 belonging to the group of collagenases, MMP-2 and MMP-9 gelatinases, MMP-3 stromelysins, MMP-7 matrilysins, MMP- 
Table 1. Functional significance of SNPs in MMPs

\begin{tabular}{|c|c|c|c|c|}
\hline Gene & dbSNP & Polymorphism & Gene region & Functional significance \\
\hline MMP-1 & rs1799750 & $-16071 \mathrm{G} / 2 \mathrm{G}$ & Promoter & $G$ insertion generates higher levels of gene transcription \\
\hline MMP-2 & rs 243865 & $-1306 \mathrm{C} / \mathrm{T}$ & Promoter & $C$ allele possesses higher transcriptional activity \\
\hline MMP-3 & rs3025058 & $-11715 \mathrm{~A} / 6 \mathrm{~A}$ & Promoter & A deletion generates higher levels of gene transcription \\
\hline MMP-7 & rs11568818 & $-181 \mathrm{~A} / \mathrm{G}$ & Promoter & $G$ allele possesses higher transcriptional activity \\
\hline MMP-9 & rs3918242 & $-1562 \mathrm{C} / \mathrm{T}$ & Promoter & $T$ allele possesses higher transcriptional activity \\
\hline \multirow[b]{2}{*}{ MMP-12 } & rs2276109 & $-82 \mathrm{~A} / \mathrm{G}$ & Promoter & $A$ allele possesses higher transcriptional activity \\
\hline & rs652438 & $1082 \mathrm{~A} / \mathrm{G}$ & Exon & $\begin{array}{c}\text { Substitution } A / G \text { results in change Asn357Ser. The functional significance has not } \\
\text { been determined }\end{array}$ \\
\hline MMP-13 & rs2252070 & $-77 \mathrm{~A} / \mathrm{G}$ & Promoter & Substitution $A / G$ generates higher levels of gene transcription \\
\hline MMP-21 & rs10901425 & $572 \mathrm{C} / \mathrm{T}$ & Exon & $\begin{array}{c}\text { Substitution } C / T \text { results in change Ala191Val in } \mathrm{N} \text {-terminal part of the catalytic } \\
\text { domain }\end{array}$ \\
\hline
\end{tabular}

12 and MMP-21 to the group of other MMPs. Table 2 shows results of case-control or prospective studies on genetic polymorphism of MMPs in relation to the risk of breast cancer, progression and survival.

Metalloproteinase-1. MMP-1, commonly known as collagenase-1, hydrolyzes collagen types: I, II, III, VII, X, gelatin, aggrecan, perlecan, link protein, entactin, tenascin, and other substrates [7]. Studies on cell lines have revealed that MMP-1 is responsible for the invasion and progression of breast cancer and formation of the metastases [24-26]. Polymorphism of $M M P-1$ at -1607 (rs 1799750) in the promoter region involves the insertion or deletion of guanine $(G)$ [27]. The insertion of $G$ allele affects the binding site for the E Twenty-Six specific domain transcription factor. Insertion of $G$ allele causes that the gene is transcriptionally more active than the gene with one $G$ allele [28]. Some research results indicates no relationship between this polymorphism and the risk of breast cancer, disease-free survival and clinical status of patients [29-32]. However, the results of research done by other researchers suggest an increased risk of lymph node metastases in patients with at least one $2 G$ allele (OR $=1.68,95 \% \mathrm{CI}: 1.19-2.39$ ), particularly in $2 G / 2 G$ homozygotes (OR $=2.14,95 \%$ CI: 1.24 -

Table 2. Genetic polymorphism MMPs in relation to breast cancer risk and survival

\begin{tabular}{|c|c|c|c|c|c|c|}
\hline Gene & $\begin{array}{c}\text { Polymorphism, } \\
\text { dbSNP }\end{array}$ & $\begin{array}{l}\text { Cases/ } \\
\text { Controls }\end{array}$ & Population & $\begin{array}{c}\text { Association } \\
\text { (study variables) }\end{array}$ & $\begin{array}{l}\text { Risk estimates * } \\
\text { OR or HR }(95 \% \text { CI })\end{array}$ & Study \\
\hline \multirow[t]{15}{*}{ MMP-1 } & \multirow[t]{13}{*}{$\begin{array}{c}-16071 \mathrm{G} / 2 \mathrm{G} \\
\mathrm{rs} 1799750\end{array}$} & $43 / 164$ & Italian & No (BCR) & NSD & $\begin{array}{l}\text { Biondi, } \\
2000[29]\end{array}$ \\
\hline & & $86 / 110$ & Italian & No (BCR, metastases, TNM) & NSD & $\begin{array}{l}\text { Ghilardi, } \\
2002[32]\end{array}$ \\
\hline & & \multirow[t]{2}{*}{$145 / 150$} & \multirow[t]{2}{*}{ Polish } & No (BCR, BR grading) & NSD & \multirow{2}{*}{$\begin{array}{c}\text { Przybylowska, } \\
2004 \text { [33] }\end{array}$} \\
\hline & & & & Yes $(\mathrm{LNM})$ & $\begin{array}{c}2 G \\
p<0.001\end{array}$ & \\
\hline & & \multirow[t]{3}{*}{$270 / 300$} & \multirow[t]{3}{*}{ Polish } & No (BCR) & NSD & \multirow{3}{*}{$\begin{array}{c}\text { Przybylowska, } \\
2006[34]\end{array}$} \\
\hline & & & & Yes (LNM) & $\begin{array}{c}2 G / 2 G \\
\mathrm{OR}=2.14(1.24-3.69) \\
2 G \\
\mathrm{OR}=1.68(1.19-2.39)\end{array}$ & \\
\hline & & & & Yes (BR grading) & $\begin{array}{c}2 G \\
\mathrm{OR}=2.58(1.35-4.91)\end{array}$ & \\
\hline & & $959 / 952$ & Swedish & No (BCR, OS) & NSD & $\begin{array}{c}\text { Lei, } \\
2007[30]\end{array}$ \\
\hline & & \multirow[t]{3}{*}{$221 /-$} & \multirow[t]{3}{*}{ mixed ethnicities } & Yes (OS) & $\begin{array}{c}2 G / 2 G \\
\mathrm{HR}=3.1(1.1-8.7)\end{array}$ & \multirow[t]{4}{*}{$\begin{array}{l}\text { Hughes, } \\
2007 \text { [35] }\end{array}$} \\
\hline & & & & Yes (LNM) & $\begin{array}{c}2 G / 2 G \\
\mathrm{OR}=3.9(1.7-9.4)\end{array}$ & \\
\hline & & & & No (DFS) & NSD & \\
\hline & & $143 /-$ & Caucasian & Yes (LNM) & $\begin{array}{c}2 G / 2 G \\
\mathrm{OR}=2.6(1.0-6.9)\end{array}$ & \\
\hline & & $1495 / 1437$ & \multirow[t]{3}{*}{ Chinese } & \multirow[t]{3}{*}{ No (BCR) } & \multirow[t]{3}{*}{ NSD } & \multirow{3}{*}{$\begin{array}{c}\text { Beeghly Fadiel, } \\
2009[31]\end{array}$} \\
\hline & $14 \times$ SNPs & $1062 / 1069$ & & & & \\
\hline & $8 \times$ SNPs & $1498 / 1496$ & & & & \\
\hline
\end{tabular}




\section{Table 2. Continued}

\begin{tabular}{|c|c|c|c|c|c|c|}
\hline Gene & $\begin{array}{l}\text { Polymorphism, } \\
\text { dbSNP }\end{array}$ & $\begin{array}{c}\text { Cases/ } \\
\text { Controls }\end{array}$ & Population & $\begin{array}{c}\text { Association } \\
\text { (study variables) }\end{array}$ & $\begin{array}{c}\text { Risk estimates * } \\
\text { OR or HR }(95 \% \mathrm{CI})\end{array}$ & Study \\
\hline \multirow[t]{15}{*}{ MMP-2 } & \multirow[t]{9}{*}{$\begin{array}{l}-1306 \mathrm{C} / \mathrm{T} \\
\mathrm{rs} 243865\end{array}$} & \multirow[t]{3}{*}{$251 /-$} & \multirow[t]{3}{*}{ Austrian } & Yes (tumor size) & $\begin{array}{c}T / T \\
p<0.006 \\
\end{array}$ & \multirow[t]{3}{*}{$\begin{array}{c}\text { Grieu, } \\
2004[37]\end{array}$} \\
\hline & & & & Yes (negative ER status) & $\begin{array}{c}T / T \\
p<0.002\end{array}$ & \\
\hline & & & & Yes (OS) & $\begin{array}{c}T / T \\
(59 / 70,84 \% ; p<0.001) \\
\text { among women negative ER } \\
\text { status } \\
T / T \\
(130 / 157,84 \% ; p<0.001) \\
\text { among women positive ER } \\
\text { status }\end{array}$ & \\
\hline & & \multirow[t]{2}{*}{$462 / 509$} & \multirow[t]{2}{*}{ Chinese } & Yes (BCR) & $\begin{array}{c}C / T+T / T \\
\mathrm{OR}=0.46(0.34-0.63)\end{array}$ & \multirow[t]{2}{*}{$\begin{array}{c}\text { Zhou, } \\
2004[38]\end{array}$} \\
\hline & & & & $\begin{array}{c}\text { No (clinical and histochemical } \\
\text { features) }\end{array}$ & NSD & \\
\hline & & $959 / 952$ & Swedish & No $(\mathrm{BCR}, \mathrm{OS})$ & NSD & $\begin{array}{c}\text { Lei, } \\
2007[30]\end{array}$ \\
\hline & & $89 / 100$ & South Brazilian & $\begin{array}{c}\text { No (BCR, clinical } \\
\text { and histochemical } \\
\text { features) }\end{array}$ & NSD & $\begin{array}{l}\text { Roehe, } \\
2007[41]\end{array}$ \\
\hline & & $90 / 96$ & Mexican & Yes $(\mathrm{BCR})$ & $\begin{array}{c}C / C \\
\mathrm{OR}=2.15(1.1-4.1) \\
C / C \\
\mathrm{OR}=2.66(1.04-6.96) \text { among } \\
\text { women }<50 \mathrm{y}\end{array}$ & $\begin{array}{l}\text { Delgado, } \\
2008[40]\end{array}$ \\
\hline & & $2557 / 2506$ & \multirow[t]{7}{*}{ Chinese } & \multirow[t]{3}{*}{ No (BCR) } & \multirow[t]{3}{*}{ NSD } & \multirow{7}{*}{$\begin{array}{l}\text { Beeghly Fadiel, } \\
2009 \text { [39] }\end{array}$} \\
\hline & G/A, rs11644561 & $2069 / 2080$ & & & & \\
\hline & T/G, rs11643630 & $2069 / 2080$ & & & & \\
\hline & $\mathrm{T} / \mathrm{A}, \mathrm{rs} 1005912$ & $2069 / 2080$ & & Yes (BCR) & $\begin{array}{c}T / A \\
\mathrm{OR}=1.2(1.0-1.3) \\
A / A \\
\mathrm{OR}=1.1(0.9-1.3)\end{array}$ & \\
\hline & $13 \times$ SNPs & $2069 / 2080$ & & \multirow[t]{3}{*}{ No (BCR) } & \multirow[t]{3}{*}{ NSD } & \\
\hline & $12 \times$ SNPs & $2557 / 2506$ & & & & \\
\hline & $6 \times$ SNPs & $4626 / 7092$ & & & & \\
\hline \multirow[t]{12}{*}{ MMP-3 } & \multirow[t]{10}{*}{$\begin{array}{l}-11715 \mathrm{~A} / 6 \mathrm{~A} \\
\mathrm{rs} 3025058\end{array}$} & $43 / 164$ & Italian & Yes (BCR) & $\begin{array}{c}5 A \\
p<0.005\end{array}$ & $\begin{array}{c}\text { Biondi, } \\
2000[29]\end{array}$ \\
\hline & & \multirow[t]{3}{*}{$86 / 110$} & \multirow[t]{3}{*}{ Italian } & Yes (metastases) & $\begin{array}{c}5 A \\
\mathrm{OR}=1.96(1.16-3.30) \\
\end{array}$ & \multirow[t]{3}{*}{$\begin{array}{l}\text { Ghilardi, } \\
2002[32]\end{array}$} \\
\hline & & & & Yes (BCR) & $\begin{array}{c}5 A \\
\mathrm{OR}=1.53(1.02-2.29) \\
\end{array}$ & \\
\hline & & & & No (TNM) & NSD & \\
\hline & & $246 / 182$ & $\begin{array}{l}\text { Swedish, } \\
\text { Czech }\end{array}$ & No (BCR) & NSD & $\begin{array}{c}\text { Lei, } \\
2002[43]\end{array}$ \\
\hline & & $500 / 500$ & Austrian & $\begin{array}{l}\text { No (BCR, clinical and } \\
\text { histochemical features, } \\
\text { LNM) }\end{array}$ & NSD & $\begin{array}{l}\text { Krippl, } \\
2004[42]\end{array}$ \\
\hline & & $221 /-$ & $\begin{array}{c}\text { mixed } \\
\text { ethnicities }\end{array}$ & No (LNM, OS, DFS) & NSD & \multirow[t]{2}{*}{$\begin{array}{l}\text { Hughes, } \\
2007[35]\end{array}$} \\
\hline & & $143 /-$ & Caucasian & No (LNM) & NSD & \\
\hline & & $959 / 952$ & Swedish & No (BCR, OS) & NSD & $\begin{array}{c}\text { Lei, } \\
2007[30]\end{array}$ \\
\hline & & $1495 / 1437$ & \multirow[t]{3}{*}{ Chinese } & \multirow[t]{3}{*}{ No (BCR) } & \multirow[t]{3}{*}{ NSD } & \multirow{3}{*}{$\begin{array}{c}\text { Beeghly Fadiel, } \\
2009[31]\end{array}$} \\
\hline & $6 \times$ SNPs & $1062 / 1069$ & & & & \\
\hline & $9 \times$ SNPs & $1498 / 1496$ & & & & \\
\hline
\end{tabular}


Table 2. Continued

\begin{tabular}{|c|c|c|c|c|c|c|}
\hline Gene & $\begin{array}{l}\text { Polymorphism, } \\
\text { dbSNP }\end{array}$ & $\begin{array}{l}\text { Cases/ } \\
\text { Controls }\end{array}$ & Population & $\begin{array}{c}\text { Association } \\
\text { (study variables) }\end{array}$ & $\begin{array}{c}\text { Risk estimates * } \\
\text { OR or HR }(95 \% \mathrm{CI})\end{array}$ & Study \\
\hline \multirow[t]{27}{*}{ MMP-7 } & \multirow{5}{*}{$\begin{array}{c}-181 \mathrm{~A} / \mathrm{G} \\
\mathrm{rs} 11568818\end{array}$} & $221 /-$ & mixed ethnicities & No (LNM, OS, DFS) & NSD & Hughes, \\
\hline & & $143 /-$ & Caucasian & No (LNM) & NSD & $2007[35]$ \\
\hline & & $1079 / 1082$ & Chinese & No (BCR) & NSD & $\begin{array}{c}\text { Beeghly Fadiel, } \\
2008[46]\end{array}$ \\
\hline & & \multirow[t]{2}{*}{$\sim 1050 /-$} & \multirow[t]{2}{*}{ Chinese } & Yes (OS) & $\begin{array}{c}G / \mathrm{G} \\
\mathrm{HR}=6.7(2.4-18.6)\end{array}$ & \multirow[t]{2}{*}{$\begin{array}{c}\text { Beeghly Fadiel } \\
2009 \text { [44] }\end{array}$} \\
\hline & & & & Yes (DFS) & $\begin{array}{c}G / \mathrm{G} \\
\mathrm{HR}=5.2(1.9-14.4)\end{array}$ & \\
\hline & \multirow[t]{3}{*}{$\mathrm{C} / \mathrm{T}, \mathrm{rs} 12184413$} & $1079 / 1082$ & Chinese & Yes (BCR) & $\begin{array}{c}T / T \\
\text { OR }=0.7(0.6-0.9) \\
T / T \\
\text { OR }=0.6(0.4-0.8) \text { among } \\
\text { post-menopausal women }\end{array}$ & $\begin{array}{c}\text { Beeghly Fadiel, } \\
2008 \text { [46] }\end{array}$ \\
\hline & & \multirow[t]{2}{*}{$\sim 1050 /-$} & \multirow[t]{2}{*}{ Chinese } & Yes (DFS) & $\begin{array}{c}T / T \\
\mathrm{HR}=0.5(0.3-0.9)\end{array}$ & \multirow[t]{2}{*}{$\begin{array}{c}\text { Beeghly Fadiel } \\
2009 \text { [44] }\end{array}$} \\
\hline & & & & Yes (OS) & $\begin{array}{c}T / T \\
\mathrm{HR}=0.4(0.2-0.9)\end{array}$ & \\
\hline & \multirow[t]{2}{*}{ A/G, rs10895304 } & $1079 / 1082$ & Chinese & Yes (BCR) & $\begin{array}{c}G \\
\mathrm{OR}=1.9(1.2-3.0) \text { among pre- } \\
\text { menopausal women }\end{array}$ & $\begin{array}{c}\text { Beeghly Fadiel, } \\
2008 \text { [46] }\end{array}$ \\
\hline & & $\sim 1050 /-$ & Chinese & No (OS, DFS) & NSD & $\begin{array}{c}\text { Beeghly Fadiel, } \\
2009[44]\end{array}$ \\
\hline & \multirow[t]{2}{*}{ T/C, rs7935378 } & $1079 / 1082$ & Chinese & Yes (BCR) & $\begin{array}{c}C \\
\text { OR }=1.9(1.2-3.0) \text { among pre- } \\
\text { menopausal women }\end{array}$ & $\begin{array}{c}\text { Beeghly Fadiel, } \\
2008 \text { [46] }\end{array}$ \\
\hline & & $\sim 1050 /-$ & Chinese & No (OS, DFS) & NSD & $\begin{array}{c}\text { Beeghly Fadiel, } \\
2009 \text { [44] }\end{array}$ \\
\hline & \multirow[t]{2}{*}{$\mathrm{A} / \mathrm{T}, \mathrm{rs} 880197$} & $1079 / 1082$ & Chinese & No (BCR) & NSD & $\begin{array}{c}\text { Beeghly Fadiel, } \\
2008 \text { [46] }\end{array}$ \\
\hline & & $\sim 1050 /-$ & Chinese & No (OS, DFS) & NSD & $\begin{array}{c}\text { Beeghly Fadiel, } \\
2009 \text { [44] }\end{array}$ \\
\hline & \multirow[t]{3}{*}{ G/A, rs17098318 } & $1079 / 1082$ & Chinese & No (BCR) & NSD & $\begin{array}{c}\text { Beeghly Fadiel, } \\
2008 \text { [46] }\end{array}$ \\
\hline & & \multirow[t]{2}{*}{$\sim 1050 /-$} & \multirow[t]{2}{*}{ Chinese } & Yes (DFS) & $\begin{array}{c}A / A \\
\mathrm{HR}=4.2(1.3-13.4)\end{array}$ & \multirow[t]{2}{*}{$\begin{array}{c}\text { Beeghly Fadiel } \\
2009[44]\end{array}$} \\
\hline & & & & Yes (OS) & $\begin{array}{c}A / A \\
\mathrm{HR}=7.0(2.2-22.8)\end{array}$ & \\
\hline & \multirow[t]{3}{*}{ A/G, rs11225307 } & $1079 / 1082$ & Chinese & No (BCR) & NSD & $\begin{array}{c}\text { Beeghly Fadiel, } \\
2008 \text { [46] }\end{array}$ \\
\hline & & \multirow[t]{2}{*}{$\sim 1050 /-$} & \multirow[t]{2}{*}{ Chinese } & Yes (DFS) & $\begin{array}{c}G / G \\
\mathrm{HR}=0.5(0.3-0.9)\end{array}$ & \multirow[t]{2}{*}{$\begin{array}{c}\text { Beeghly Fadiel } \\
2009 \text { [44] }\end{array}$} \\
\hline & & & & No (OS) & NSD & \\
\hline & \multirow[t]{3}{*}{$\mathrm{A} / \mathrm{T}, \mathrm{rs} 11225297$} & $1079 / 1082$ & Chinese & No (BCR) & NSD & $\begin{array}{c}\text { Beeghly Fadiel, } \\
2008 \text { [46] }\end{array}$ \\
\hline & & $\sim 1050 /-$ & Chinese & Yes (OS) & $\begin{array}{c}A / T \\
\mathrm{HR}=0.7(0.5-0.9) \\
T / T \\
\mathrm{HR}=0.3(0.1-0.8) \\
\end{array}$ & $\begin{array}{c}\text { Beeghly Fadiel, } \\
2009 \text { [44] }\end{array}$ \\
\hline & & & & Yes (DFS) & $\begin{array}{c}T / T \\
\mathrm{HR}=0.4(0.2-0.8)\end{array}$ & \\
\hline & \multirow[t]{2}{*}{$\mathrm{A} / \mathrm{C}, \mathrm{rs} 17352054$} & $1079 / 1082$ & Chinese & No (BCR) & NSD & $\begin{array}{c}\text { Beeghly Fadiel, } \\
2008 \text { [46] }\end{array}$ \\
\hline & & $\sim 1050 /-$ & Chinese & No (OS, DFS) & NSD & $\begin{array}{c}\text { Beeghly Fadiel, } \\
2009 \text { [44] }\end{array}$ \\
\hline & \multirow[t]{2}{*}{$\mathrm{C} / \mathrm{T}, \mathrm{rs} 495041$} & $1079 / 1082$ & Chinese & Yes (BCR) & $\begin{array}{c}C / T \\
\mathrm{HR}=1.6(1.1-2.3) \text { among } \\
\text { post-menopausal women }\end{array}$ & $\begin{array}{c}\text { Beeghly Fadiel, } \\
2008 \text { [46] }\end{array}$ \\
\hline & & $\sim 1050 /-$ & Chinese & No (OS, DFS) & NSD & $\begin{array}{c}\text { Beeghly Fadiel, } \\
2009 \text { [44] }\end{array}$ \\
\hline
\end{tabular}


Table 2. Continued

\begin{tabular}{|c|c|c|c|c|c|c|}
\hline Gene & $\begin{array}{l}\text { Polymorphism, } \\
\text { dbSNP }\end{array}$ & $\begin{array}{c}\text { Cases/ } \\
\text { Controls }\end{array}$ & Population & $\begin{array}{c}\text { Association } \\
\text { (study variables) }\end{array}$ & $\begin{array}{c}\text { Risk estimates * } \\
\text { OR or HR }(95 \% \mathrm{CI})\end{array}$ & Study \\
\hline \multirow[t]{7}{*}{ MMP-8 } & $\begin{array}{c}\mathrm{A} / \mathrm{G}, \mathrm{rs} 10895353 \\
\mathrm{C} / \mathrm{T}, \mathrm{rs} 7943404 \\
\mathrm{C} / \mathrm{T}, \mathrm{rs} 2508383 \\
\mathrm{~A} / \mathrm{G}, \mathrm{rs} 1320632 \\
\mathrm{~A} / \mathrm{T}, \mathrm{rs} 17099436 \\
\mathrm{~A} / \mathrm{G}, \mathrm{rs} 1940475 \\
\mathrm{~A} / \mathrm{T}, \mathrm{rs} 1892986\end{array}$ & $140 /-$ & Belgium & $\begin{array}{l}\text { No (clinical and histo- } \\
\text { chemical features, LNM) }\end{array}$ & NSD & $\begin{array}{l}\text { Decock, } \\
2007[50]\end{array}$ \\
\hline & \multirow[t]{2}{*}{$\mathrm{A} / \mathrm{G}, \mathrm{rs} 1276284$} & \multirow[t]{2}{*}{$140 /-$} & \multirow[t]{2}{*}{ Belgium } & $\begin{array}{c}\text { No (clinical and } \\
\text { histochemical features) }\end{array}$ & NSD & \\
\hline & & & & Yes $(\mathrm{LNM})$ & $\begin{array}{c}G \\
\mathrm{OR}=0.6(0.3-0.9)\end{array}$ & \\
\hline & \multirow[t]{4}{*}{$\mathrm{C} / \mathrm{T}, \mathrm{rs} 11225395$} & \multirow[t]{2}{*}{$140 /-$} & \multirow[t]{2}{*}{ Belgium } & $\begin{array}{c}\text { No (clinical and } \\
\text { histochemical features) }\end{array}$ & NSD & \\
\hline & & & & Yes $(\mathrm{LNM})$ & $\begin{array}{c}T \\
\mathrm{OR}=0.6(0.4-0.9)\end{array}$ & \\
\hline & & \multirow[t]{2}{*}{$\sim 1100 /-$} & \multirow[t]{2}{*}{ Chinese } & $\begin{array}{c}\text { No (clinical and } \\
\text { histochemical features, OS) }\end{array}$ & NSD & \\
\hline & & & & Yes (DFS) & $\begin{array}{c}T \\
\mathrm{HR}=0.7(0.5-0.9) \text { among pa- } \\
\text { tients with earlier stage cancer }\end{array}$ & \\
\hline \multirow[t]{15}{*}{ MMP-9 } & \multirow[t]{12}{*}{$\begin{array}{l}-1562 \mathrm{C} / \mathrm{T} \\
\mathrm{rs} 3918242\end{array}$} & \multirow[t]{4}{*}{$251 /-$} & \multirow[t]{4}{*}{ Austrian } & Yes (non ductal tumor type) & $\begin{array}{c}T \\
p<0.02\end{array}$ & \multirow[t]{4}{*}{$\begin{array}{c}\text { Grieu, } \\
2004[37]\end{array}$} \\
\hline & & & & Yes (positive ER status) & $p<0.033$ & \\
\hline & & & & $\begin{array}{c}\text { Yes (absence of present } p 53 \\
\text { mutation) }\end{array}$ & $\begin{array}{c}T \\
p<0.025\end{array}$ & \\
\hline & & & & Yes $(\mathrm{OS})$ & $\begin{array}{c}C / T \\
p<0.06\end{array}$ & \\
\hline & & \multirow[t]{2}{*}{$270 / 300$} & \multirow[t]{2}{*}{ Polish } & Yes (BR grading) & $\begin{array}{c}T \\
\mathrm{OR}=2.61(1.33-4.87)\end{array}$ & \multirow[t]{2}{*}{$\begin{array}{c}\text { Przybylowska, } 2006 \\
{[34]}\end{array}$} \\
\hline & & & & No (BCR) & NSD & \\
\hline & & \multirow[t]{2}{*}{$221 /-$} & \multirow[t]{2}{*}{ mixed ethnicities } & Yes (LNM) & $\begin{array}{c}C / T \\
\mathrm{OR}=3.6(1.2-11.1)\end{array}$ & \multirow[t]{3}{*}{$\begin{array}{l}\text { Hughes, } \\
2007[35]\end{array}$} \\
\hline & & & & No (OS, DFS) & NSD & \\
\hline & & $143 /-$ & Caucasian & Yes (LNM) & $\begin{array}{c}C / T \\
\mathrm{OR}=9.1(1.7-48.4) \\
\end{array}$ & \\
\hline & & \multirow[t]{2}{*}{$959 / 952$} & \multirow[t]{2}{*}{ Swedish } & Yes (BCR) & $\begin{array}{c}T / T \\
\mathrm{OR}=1.88(0.97-3.63)\end{array}$ & \multirow[t]{2}{*}{$\begin{array}{c}\text { Lei, } \\
2007[30]\end{array}$} \\
\hline & & & & No $(\mathrm{OS})$ & NSD & \\
\hline & & $96 / 100$ & South Brazilian & $\begin{array}{l}\text { No (BCR, clinical and } \\
\text { histochemical features) }\end{array}$ & NSD & $\begin{array}{c}\text { Roehe, } \\
2007[41]\end{array}$ \\
\hline & $\mathrm{T} / \mathrm{A}, \mathrm{rs} 3918241$ & $5408 / 2899$ & \multirow[t]{3}{*}{ Chinese } & \multirow[t]{3}{*}{ No (BCR) } & \multirow[t]{3}{*}{ NSD } & \multirow{3}{*}{$\begin{array}{l}\text { Beeghly Fadiel, } \\
2011 \text { [52] }\end{array}$} \\
\hline & $\begin{array}{c}\text { G/A, rs2274756 } \\
\text { C/T, rs4810482 } \\
\text { G/A, rs } 17576 \\
\mathrm{C} / \mathrm{G}, \mathrm{rs} 2250889\end{array}$ & $\sim 1058 / \sim 1063$ & & & & \\
\hline & $\begin{array}{l}\mathrm{T} / \mathrm{C}, \mathrm{rs} 3918249 \\
\mathrm{~A} / \mathrm{G}, \mathrm{rs} 6065912\end{array}$ & $\sim 2000 / \sim 2000$ & & & & \\
\hline \multirow[t]{4}{*}{ MMP-12 } & \multirow[t]{3}{*}{$\begin{array}{l}-82 \mathrm{~A} / \mathrm{G} \\
\mathrm{rs} 2276109\end{array}$} & $1129 / 1129$ & Chinese & $\begin{array}{l}\text { No (BCR, clinical and histo- } \\
\text { chemical features, OS, DFS) }\end{array}$ & NSD & $\begin{array}{c}\text { Shin, } \\
2005[54]\end{array}$ \\
\hline & & $221 /-$ & mixed ethnicities & No (LNM, OS, DFS) & NSD & Hughes, \\
\hline & & $143 /-$ & Caucasian & No (LNM) & NSD & $2007[35]$ \\
\hline & $\begin{array}{l}1082 \mathrm{~A} / \mathrm{G} \\
\mathrm{rs} 652438\end{array}$ & $1129 / 1129$ & Chinese & $\begin{array}{l}\text { No (BCR, clinical and histo- } \\
\text { chemical features, OS, DFS) }\end{array}$ & NSD & $\begin{array}{c}\text { Shin, } \\
2005[54]\end{array}$ \\
\hline \multirow[t]{3}{*}{ MMP-13 } & $\begin{array}{c}-77 \mathrm{~A} / \mathrm{G} \\
\mathrm{rs} 2252070\end{array}$ & $959 / 952$ & Swedish & No $(\mathrm{BCR}, \mathrm{OS})$ & NSD & $\begin{array}{c}\text { Lei, } \\
2007[30]\end{array}$ \\
\hline & & $221 /-$ & mixed ethnicities & No (LNM, OS, DFS) & NSD & Hughes, \\
\hline & & $143 /-$ & Caucasian & No (LNM) & NSD & $2007[35]$ \\
\hline MMP-21 & $\begin{array}{c}572 \mathrm{C} / \mathrm{T}, \\
\mathrm{rs} 10901425\end{array}$ & $76 / 320$ & Russian & $\begin{array}{c}\text { No (BCR, clinical and histo- } \\
\text { chemical features) }\end{array}$ & NSD & $\begin{array}{l}\text { Shagisultanova, } \\
2004[56]\end{array}$ \\
\hline
\end{tabular}

Studies that have associated with BCR, LNM, TNM, ER status, BR grading, OS or DFS have been noted in bold

${ }^{*}$ The reference is the common homozygote genotype or common allele

Abbreviations: BCR, breast cancer risk; LNM, lymph node metastasis; TNM, tumor, nodus, metastasis; ER, estrogen receptor status; BR, Bloom-Richardson grading; OS, overall survival; DFS, disease-free survival; HR, hazard ratio; OR, odds ratio; NSD, no significant difference 
3.69) $[33,34]$. Research conducted in 2007 by Hughes et al. confirmed the increased risk of lymph node metastases in $2 \mathrm{G} / 2 \mathrm{G}$ homozygotes (OR $=3.9,95 \% \mathrm{CI}: 1.7-9.4$ ) [35].

Metalloproteinase-2. MMP-2, also known as gelatinase A or collagenase IV, uses collagen types: I, IV, V, VII, X, XI, elastin, gelatin fibronectin, laminin-5, brevican, neurocan, decorin, BM-40, vitronectin, aggrecan, and other substrates [7]. In an in vitro experiment, it was noted that the polymorphism at $-1306 \mathrm{C} / \mathrm{T}$ ( $r s 243865)$ affects gene expression of $M M P-2$. Transcriptional activity of $M M P-2$ with the thymine $(T)$ allele is lower than the cytosine $(C)$ allele [36]. It was suggested, that $C / T$ substitution abolish Sp1 transcription factor binding site in the $M M P-2$ gene $[36,37]$. The results of the survey conducted among the Chinese population by Zhou and colleagues suggest a reduced risk of breast cancer for $C / T$ and $T / T$ genotype $(\mathrm{OR}=0.46,95 \% \mathrm{CI}: 0.34-0.63$ ) [38]. On the other hand, Beeghly-Fadiel et al. reported a tendency to increased risk of breast cancer in $T / T$ homozygotes $(\mathrm{OR}=1.4,95 \% \mathrm{CI}$ : 0.9-2.4) [39]. Delgado et al. have demonstrated high risk of developing breast cancer for the $C / C$ genotype $(\mathrm{OR}=2.15$, 95\% CI: 1.1-4.1) [40]. Test results from other centers do not show a relationship between this polymorphism and the risk of breast cancer [30, 41]. For that MMP, other SNPs showing a relationship of polymorphism with the risk of breast cancer for $r s 1005912(\mathrm{OR}=1.2,95 \% \mathrm{CI}: 1.0-1.3), r s 11644561(\mathrm{OR}=$ $0.6,95 \%$ CI: 0.3-1.0), $r s 11643630$ (OR = 0.8, 95\% CI: 0.7-1.0) have been also tested. Analysis of 32 other SNPs showed no relationship with the risk of breast cancer [39].

Metalloproteinase-3. MMP-3, known also as stromelysin1 , catalyzes the hydrolysis of collagen types: II, III, IV, V, IX, $\mathrm{X}, \mathrm{XI}$, gelatin, fibronectin, vitronectin, laminin, entactin, tenascin, elastin, aggrecan, link protein, perlecan, decorin, fibrin/fibrinogen, and other substrates [7]. Polymorphism of $M M P-3$ gene involving insertion or deletion of adenine $(A)$ at -1171 5A/6A (rs3025058), affects the strength of transcription factor binding and promoter activity [42]. Ye and colleagues in their in vitro study found that $5 \mathrm{~A}$ allele is characterized by about 2 -fold higher promoter activity than $6 \mathrm{~A}$ allele [19]. The first study of this SNP reported elevated risk of breast cancer in women with $5 A$ allele $(p<0.005)$ [29], but this has not been confirmed by other studies [30,31, 42, 43]. The next study report a relationship between $M M P-35 A / 5 A$ genotype and the frequency of metastases ( $\mathrm{OR}=1.96,95 \%$ CI: 1.16-3.30) [32]. No relationship of this polymorphism with the length of survival in breast cancer has been proven to occur $[30,35]$.

Metalloproteinase-7. MMP-7, known also as matrilysin-1 or PUMP-1, is an enzyme hydrolyzing the ECM components, such as aggrecan, entactin, laminin, vitronectin, fibrin/fibrinogen, tenascin, gelatin, fibronectin, collagen types: IV, V, IX, X, XI, and other substrates [7]. A/G substitution at -181 (rs11568818) affects the transcriptional activity of $M M P-7$ gene and leads to changes in the expression of this gene. So the $G$ allele may cause an increase in gene transcription, and thus increase enzyme activity [45]. The first study of a diverse group of breast cancer patients, predominantly Caucasians, showed no relationship between the substitution $A / G$, and lymph node metastasis and survival [35]. Beeghly-Fadiel et al. studying a Chinese population confirmed no association with the risk of breast cancer for this polymorphism and six other ones in the gene encoding this enzyme. At the same time a significant relationship between cancer risk and the $r s 12184413 \mathrm{~T} / \mathrm{T}$ genotype (OR $=0.7,95 \%$ CI: 0.6-0.9) and $r s 10895304 G$ allele (OR $=1.9,95 \%$ CI: 1.2-3.0), rs7935378 C allele $(\mathrm{OR}=1.9,95 \% \mathrm{CI}$ : 1.2-3.0) has been shown to occur, but only for pre-menopausal women [46]. In further studies, Beeghly-Fadiel et al. showed a significant correlation between the survival time of women with breast cancer and the $r s 11568818 \mathrm{G}$ allele $(\mathrm{HR}=6.7,95 \%$ CI: 2.4-18.6), $r s 11225297 \mathrm{~A} / \mathrm{T}$ genotype $(\mathrm{HR}=0.7,95 \% \mathrm{CI}$ : $0.5-0.9)$ and $T / T(\mathrm{HR}=0.3,95 \% \mathrm{CI}: 0.1-0.8), r s 11225307 \mathrm{G} / \mathrm{G}$ $(\mathrm{HR}=0.5,95 \% \mathrm{CI}: 0.3-0.9), r s 12184413 \mathrm{~T} / \mathrm{T}(\mathrm{HR}=0.5,95 \%$ CI: $0.3-0.9), r s 17098318 A / A$ (HR $=4.2,95 \%$ CI: $1.3-13.4)$. For the other SNPs of this gene, $r s 17352054, r s 495041$, rs 10895304 , $r s 7935378$, rs 880197 but showed no dependence with the course of the disease [44].

Metalloproteinase-8. MMP-8, or collagenase-2, hydrolyzes collagen types: I, II, III, VII, X, gelatin, entactin, tenascin, aggrecan, and other substrates [7]. Studies on the susceptibility to skin cancer conducted in mice showed that MMP-8, paradoxically, has a protective role, since the absence of the $M M P-8$ gene resulted in an increased incidence of skin tumors in male mice [47]. This result was confirmed in the study on human breast cancer cells, where increased expression of that MMP resulted in lower tumor invasiveness [48]. These reports and the finding that the SNP of $M M P-8$ gene affects the expression of MMP-8 [49], aroused the interest of Decock et al. who in 2007 presented the results for nine different SNPs, selected from the HapMap database. The studies showed no association between the SNPs and the tumor size, the histologic grade or subtype of breast cancer. However, a correlation has been shown to occur between lymph node metastasis and four SNPs $r s 1940475 G$ allele $(p=0.03), r s 1892986 A$ allele $(p=0.03), r s 1276284 A$ allele $(p=0.03), r s 11225395 T$ allele $(p=0.02)$. Decock et al. also using blood samples from breast cancer patients from an extensive database (Shanghai Breast Cancer Study) have confirmed that the MMP-8 (rs11225395) with $T$ allele is associated with lower frequency of metastasis to lymph nodes compared with $C$ allele and with longer survival of patients with early stage cancer [50].

Metalloproteinase-9. MMP-9, commonly referred to as gelatinase B, hydrolyzes gelatin, collagen types: I, IV, V, VII, $\mathrm{X}, \mathrm{XI}$, elastin, fibronectin, vitronectin, aggrecan, link protein, laminin, and other substrates [7]. There are indications that the polymorphism of $M M P-9$ at $-1562 C / T(r s 3918242)$ affects gene expression of MMP-9 and $T$ allele is associated with a 1.5 -fold higher transcriptional activity compared with the $C$ allele [51]. Grieu et al. in 2004 were the first to show a possible $(p<0.06)$ association of $C / T$ and $T / T$ genotypes with a better prognosis of patients with breast cancer compared with the $C / C$ genotype [37]. The results of Hughes et al. indicate a relationship between the risk of metastasis and $\mathrm{C} / \mathrm{T}$ genotype $(\mathrm{OR}=3.6$, 
95\% CI: 1.2-11.1) [35]. However Przybylowska et al. showed that the $T$ allele is associated with high risk of increased malignancy of breast cancer $(\mathrm{OR}=2.61,95 \% \mathrm{CI}: 1.33-4.87)$ [34]. Lei et al. studies show no effect of genotype on the increased risk of breast cancer and subsequent prognosis of survival for the $T / T$ genotype [30]. Roehe and colleagues who have examined this polymorphism, in turn, report no dependence between the risk and clinicopathological features of breast cancer [41]. Beeghly-Fadiel and colleagues also studied other SNPs for $M M P-9$ gene, but their results do not indicate a relationship with the risk of breast cancer [52].

Metalloproteinase-12. MMP-12 is also called macrophage elastase or metalloelastase. It uses elastin, laminin, fibronectin, fibrin/fibrinogen, proteoglycan, and other substrates [7]. Analysis of the results of in vitro strudies demonstrates that $M M P-12$ polymorphism at -82 which consists in the substitution $A / G$, present in the promoter region, is associated with increased transcriptional activity for the $A$ allele, because this allele affects the binding of transcription factor AP-1 [53]. The studies of polymorphism in that metalloproteinase did not show, however, an association with the risk of breast cancer with clinical stage [54], metastases to lymph nodes [35] or patient survival $[35,54]$. For that MMP, $A / G$ polymorphism at 1082 ( $r$ 652438) has been tested, which is located in the coding region of the hemopexin domain. As a result of substitution of $A / G$, asparagine (Asn) is changed to serine (Ser) in an amino acid in codon 357 . Functional significance of this polymorphism has not been clearly explained. There was no link between this polymorphism and risk of developing breast cancer. However, an association has been confirmed with the time of survival of women with breast cancer. $M M P-12 \mathrm{~A} / \mathrm{G}$ or $G / G$ genotype gave poor survival prognosis, compared to $A / A$ genotype ( $\mathrm{HR}=1.36,95 \% \mathrm{CI}$ : 0.92-2.00) [54].

Metalloproteinase-13. MMP-13 has a common name collagenase-3 and uses collagen types: I, II, III, VII, X, gelatin, aggrecan, tenascin, entactin, and other substrates [7]. It has been shown that the $A / G$ substitution at -77 ( $r$ s2252070) makes the gene transcriptionally activated, which brings with it increased activity of enzyme. In 2007, Hughes and colleagues studied patients with breast cancer in whom there was no association between the polymorphism and lymph node metastases or survival [35]. Also Lei et al., based on case-control study, showed no significant link between this polymorphism and the risk of developing breast cancer or survival time [30].

Metalloproteinase-21. MMP-21 also called XMMP, is the proteinase hydrolyzing gelatin [7]. Polymorphism at 572 ( $r$ s10901425) associated with the $C / T$ substitution in this gene leads to an amino acid change from alanine (Ala) to valine (Val) in codon 191 of the catalytic domain [55]. In a study conducted in 2004 by Shagisultanova et al. among healthy women and breast cancer patients, there was no link between this polymorphism progression and the development of the breast cancer [56].

Conclusions. The first study on genetic polymorphism of MMPs in women with breast cancer was conducted in 2000.
Since then, polymorphisms of MMPs belonging to nine different groups were analyzed among breast cancer patients of different ethnicity, and the results were published in 19 papers. Based on published results of these studies, four meta-analyses have been prepared [57-60]. Those analyses were combined with the results of individual studies in order to increase statistical power and exclude the false positive or negative results. The results of the meta-analysis published by McColgan et al. point to a lack of association between the risk of breast cancer and the MMP-1 -1607 1G/2G (rs1799750), MMP-2 -1306C/T (rs243865), MMP-3 -1171 5A/6A (rs3025058) and MMP-9 $-1562 C / T$ ( $r$ 3918242) polymorphisms. Estimated OR for MMP-1 1G/2G heterozygotes was $1.6195 \%$ CI: $0.76-1.77 ; p=$ 0.49 and $(\mathrm{OR}=1.08,95 \%$ CI: $0.66-1.76 ; p=0.75)$ for $2 G / 2 G$ variant heterozygotes, compared against $1 \mathrm{G} / 1 \mathrm{G}$ common genotype. $M M P-2 C / T$ genotype when compared against the $C / C(\mathrm{OR}=0.66,95 \%$ CI: $0.40-1.07 ; p=0.09)$ or $T / T$ genotype when compared against the $C / T$ and $T / T$ (OR $=0.95,95 \%$ CI: $0.54-1.66 ; p=0.86$ ) was not related with breast cancer risk. Similarly, this meta-analysis showed lack of association between breast cancer risk and $M M P-3$ common $5 \mathrm{~A} / 5 \mathrm{~A}$ genotype $(\mathrm{OR}=0.94,95 \% \mathrm{CI}: 0.53-1.65 ; p=0.83)$ or rare $6 \mathrm{~A} / 6 \mathrm{~A}$ genotype $(\mathrm{OR}=0.71,95 \% \mathrm{CI}: 0.38-1.33 ; p=0.29)$ and $M M P-9$ heterozygote $(\mathrm{OR}=1.07,95 \% \mathrm{CI}: 0.88-1.30 ; p=0.50)$ or $\mathrm{T} / \mathrm{T}$ genotype $(\mathrm{OR}=1.03,95 \% \mathrm{CI}: 0.16-6.75 ; p=0.98)$ [59]. These conclusions were confirmed in two other meta-analyses by Peng et al., where it was found that those polymorphisms are not likely to be a major risk factor for breast cancer, but should be taken into account because the risk may be increased as a result of exposure to certain environmental factors $[57,58]$. On the other hand, Zhou and colleagues studying the same four polymorphisms, demonstrated for $M M P-2-1306 C / T$ ( $r s 243865)$ a significantly high risk of breast cancer associated with $C / C$ genotype $(\mathrm{OR}=1.27,95 \% \mathrm{CI}: 1.10-1.47 ; p=$ 0.001 ), and a significantly low risk of breast cancer associated with $C / T$ genotype $(\mathrm{OR}=0.78,95 \% \mathrm{CI}: 0.67-0.91 ; p=0.001)$. They demonstrated no significant difference for the remaining of the analyzed polymorphisms (MMP-1 $-16071 G / 2 G$, $M M P-3-11715 A / 6 A, M M P-9-1562 C / T)$. The authors of that study conclude that MMP-2 (rs243865) polymorphism may contribute to better assessment of the risk of breast cancer [60]. However, the results obtained in this meta-analysis have been received by other scientists as unreliable and not convincing. This is evidenced by two letters to the editor by $\mathrm{He}$ [61] and Wu et al. [62], in which the authors commented on this meta-analysis. They suggest to perform a new more accurate estimation in meta-analysis of MMPs polymorphism and cancer risk. Moreover, there is also a necessity to perform a pooled-analysis.

Recent genome-wide association studies (GWAS), where hundreds of thousands of SNPs are genotyped simultaneously in very large study populations, have identified several risk alleles to be associated with breast cancer. Most of these studies were conducted in women of European ancestry, but also in Chinese and Ashkenazi Jewish populations. Although GWAS 
led to the identification of unique breast cancer susceptibility loci and confirmed previously reported ones, MMPs variants do not seem to be breast cancer genetic markers [63].

MMPs display characteristics responsible for the processes of tumor vascularization (proangiogenic activity), thereby contributing to local tumor growth and formation of metastases and, therefore, seem to be a good target in the search for new factors in the etiology and treatment of cancer in general and breast cancer in particular. However, the complexity of the interoperable system of different types of cells in the intercellular space, numerous metabolic pathways, the presence of inhibitors of MMPs and growth factors, leading to activation or inhibition of MMPs make it very difficult to determine unambiguously their role. Besides, they are combined into a network of interdependencies and, therefore, overexpression of one of MMPs may cause changes in the expression or activity of other MMPs.

Data collected in this work confirm the impact of various genetic polymorphisms of MMPs on breast cancer risk, and also on the prognosis of women with breast cancer. Unfortunately, none of the reported studies has shown that any of the nine described polymorphisms of MMPs may serve as a genetic marker to assess the risk of developing breast cancer or its further progression.

As the impact of the known mechanism of action of functional polymorphisms of MMPs in specific types of breast cancer in women is not clear, it is believed that further study of these enzymes' SNPs are inevitable and necessary. Therefore, valuable research, in addition to determine the risk of breast cancer, should also consider additional factors, such as ethnicity, gene-gene and gene-environment interactions. The influence of some polymorphisms on the risk of cancer is often observable only in combination or as a result of interaction with environmental factors, because cancer is a disease that involves many factors and mechanisms. To create an effective tool for early cancer detection and prognosis, it is necessary to extend our understanding of its etiology and explore new mechanisms, such as those affecting the transcriptional activity of MMPs genes, and thus the process of tumor progression. Perhaps studying the relationship between SNPs in one or more MMPs that have a common effect on the risk of developing cancer and its further progression, i.e. evaluation of MMPs haplotypes, will provide additional and more precise information on the role MMPs in the development of breast cancer, as well as information on their role in cancer therapy, by adjusting it to the individual's genotype pattern.

Acknowledgements: This work was supported by the internal grant: IMP 1.7/2011.

\section{References}

[1] JEMAL A, BRAY F, CENTER MM, FERLAY J, WARD E et al. Global Cancer Statistics. CA Cancer J Clin 2011;61:69-90. http://dx.doi.org/10.3322/caac.20107
[2] KELSEY JL Breast-cancer epidemiology - summary and future-directions. Epidemiol Rev 1993; 15: 256-263.

[3] ANTONIOU AC, PHAROAH PDP, MCMULLAN G, DAY NE, PONDER BAJ et al. Evidence for further breast cancer susceptibility genes in addition to BRCA1 and BRCA2 in a population-based study. Genet Epidemiol 2001; 21: 1-18. http://dx.doi.org/10.1002/gepi.1014

[4] GROSS J, LAPIERE CM Collagenolytic activity in amphibian tissues - a tissue culture assay. Proc Natl Acad Sci U S A 1962; 48: 1014-1022. http://dx.doi.org/10.1073/pnas.48.6.1014

[5] DEJONCKHEERE E, VANDENBROUCKE RE, LIBERT C Matrix metalloproteinase8 has a central role in inflammatory disorders and cancer progression. Cytokine Growth Factor Rev 2011; 22: 73-81. http://dx.doi.org/10.1016/j.cytogfr.2011.02.002

[6] VISSE R, NAGASE H Matrix metalloproteinases and tissue inhibitors of metalloproteinases - Structure, function, and biochemistry. Circ Res 2003; 92: 827-39. http://dx.doi. org/10.1161/01.RES.0000070112.80711.3D

[7] OVERALL CM Molecular determinants of metalloproteinase substrate specificity - Matrix metalloproteinase substrate binding domains, modules, and exosites. Mol Biotechnol 2002; 22: 51-86. http://dx.doi.org/10.1385/MB:22:1:051

[8] NABESHIMA K, INOUE T, SHIMAO Y, SAMESHIMA $\mathrm{T}$ Matrix metalloproteinases in tumor invasion: Role for cell migration. Pathol Int 2002; 52: 255-264. http://dx.doi. org/10.1046/j.1440-1827.2002.01343.x

[9] NAGASE H, WOESSNER JF Matrix metalloproteinases. J Biol Chem 1999; 274: 21491-21494. http://dx.doi.org/10.1074/ jbc. 274.31 .21491

[10] NGUYEN M, ARKELL J, JACKSON CJ Human endothelial gelatinases and angiogenesis. Int J Biochem Cell Biol 2001; 33: 960-970. http://dx.doi.org/10.1016/S1357-2725(01)00007-3

[11] GIALELI C, THEOCHARIS AD, KARAMANOS NK Roles of matrix metalloproteinases in cancer progression and their pharmacological targeting. FEBS J 2011; 278: 16-27. http:// dx.doi.org/10.1111/j.1742-4658.2010.07919.x

[12] RADISKY ES, RADISKY DC Matrix metalloproteinase-induced epithelial-mesenchymal transition in breast cancer. J Mammary Gland Biol Neoplasia 2010; 15: 201-212 http:// dx.doi.org/10.1007/s10911-010-9177-X

[13] EGEBLAD M, WERB Z New functions for the matrix metalloproteinases in cancer progression. Nat Rev Cancer 2002; 2: 161-174. http://dx.doi.org/10.1038/nrc745

[14] CARGill M, ALTSHULER D, IRELAND J, SKLAR P, ARDLIE $\mathrm{K}$ et al. Characterization of single-nucleotide polymorphisms in coding regions of human genes. Nat Genet 1999; 22: 231-238. http://dx.doi.org/10.1038/10290

[15] GIBBS RA, BELMONT JW, HARDENBOL P, WILLIS TD, YU FL et al. The International HapMap Project. Nature 2003; 426: 789-796. http://dx.doi.org/10.1038/nature02168

[16] CARLSON CS, EBERLE MA, KRUGLYAK L, NICKERSON DA Mapping complex disease loci in whole-genome association studies. Nature 2004; 429: 446-452. http://dx.doi. org/10.1038/nature02623

[17] BALASUBRAMANIAN SP, BROWN NJ, REED MWR Role of genetic polymorphisms in tumour angiogenesis. Br J Cancer 2002; 87: 1057-1065. http://dx.doi.org/10.1038/sj.bjc.6600625 
[18] YAN CH, BOYD DD Regulation of matrix metalloproteinase gene expression. J Cell Physiol 2007; 211: 19-26. http://dx.doi. org/10.1002/jcp.20948

[19] YE S Polymorphism in matrix metalloproteinase gene promoters: implication in regulation of gene expression and susceptibility of various diseases. Matrix Biol 2000; 19: 623629. http://dx.doi.org/10.1016/S0945-053X(00)00102-5

[20] DUFFY MJ, MAGUIRE TM, HILL A, MCDERMOTT E, O'HIGGINS N Metalloproteinases: role in breast carcinogenesis, invasion and metastasis. Breast Cancer Res 2000;2:252-257. http://dx.doi.org/10.1186/bcr65

[21] HOLLIDAY DL, BROUILETTE KT, MARKERT A, GORDON LA, JONES JL Novel multicellular organotypic models of normal and malignant breast: tools for dissecting the role of the microenvironment in breast cancer progression. Breast Cancer Res 2009; 11: 1. http://dx.doi.org/10.1186/bcr2218

[22] FANG SM, JIN X, WANG R, LI Y, GUO W et al. Polymorphisms in the MMP1 and MMP3 promoter and non-small cell lung carcinoma in North China. Carcinogenesis 2005; 26: 481-486

[23] MATSUMURA S, OUE N, NAKAYAMA H, KITADAI Y, YOSHIDA $\mathrm{K}$ et al. A single nucleotide polymorphism in the MMP-9 promoter affects tumor progression and invasive phenotype of gastric cancer. J Cancer Res Clin Oncol 2005;131:19-25. http://dx.doi.org/10.1007/s00432-004-0621-4

[24] ECK SM, HOOPES PJ, PETRELLA BL, COON CI, BRINCKERHOFF CE Matrix metalloproteinase-1 promotes breast cancer angiogenesis and osteolysis in a novel in vivo model. Breast Cancer Res Treat 2009; 116: 79-90. http://dx.doi. org/10.1007/s10549-008-0085-3

[25] LOO WTY, CHEUNG MNB, CHOW LWC Production of matrix metalloproteinases in specific subpopulations of human-patient breast cancer invading in three dimensional culture system. Life Sci 2004; 76: 743-752. http://dx.doi. org/10.1016/j.lfs.2004.06.027

[26] PARK YH, JUNG HH, AHN JS, IM YH Ets-1 upregulates HER2-induced MMP-1 expression in breast cancer cells. Biochem Biophys Res Commun 2008; 377: 389-394. http://dx.doi. org/10.1016/j.bbrc.2008.09.135

[27] RUTTER JL, MITCHELL TI, BUTTICE G, MEYERS J, GUSELLA JF et al. A single nucleotide polymorphism in the matrix metalloproteinase-1 promoter creates an Ets binding site and augments transcription. Cancer Res 1998; 58: 53215325 .

[28] TOWER GB, COON CC, BENBOW U, VINCENTI MP, BRINCKERHOFF CE Erk 1/2 differentially regulates the expression from the $1 \mathrm{G} / 2 \mathrm{G}$ single nucleotide polymorphism in the MMP-1 promoter in melanoma cells. Biochim Biophys Acta 2002; 1586: 265-274.

[29] BIONDI ML, TURRI O, LEVITI S, SEMINATI R, CECCHINI F et al. MMP1 and MMP3 polymorphisms in promoter regions and cancer. Clin Chem 2000; 46: 2023-2024.

[30] LEI HX, HEMMINKI K, ALTIERI A, JOHANSSON R, ENQUIST K et al. Promoter polymorphisms in matrix metalloproteinases and their inhibitors: few associations with breast cancer susceptibility and progression. Breast Cancer Res Treat 2007; 103: 61-69. http://dx.doi.org/10.1007/s10549-006-9345-2
[31] BEEGHLY-FADIEL A, CAI QY, LU W, LONG JR, GAO YT et al. No Association between Matrix Metalloproteinase-1 or Matrix Metalloproteinase-3 Polymorphisms and Breast Cancer Susceptibility: A Report from the Shanghai Breast Cancer Study. Cancer Epidemiol Biomarkers Prev 2009; 18: 1324-1327. http://dx.doi.org/10.1158/1055-9965.EPI-090046

[32] GHILARDI G, BIONDI ML, CAPUTO M, LEVITI S, DEMONTI $\mathrm{M}$ et al. A single nucleotide polymorphism in the matrix metalloproteinase- 3 promoter enhances breast cancer susceptibility. Clin Cancer Res 2002; 8: 3820-3823.

[33] PRZYBYLOWSKA K, ZIELINSKA J, ZADROZNY M, KRAWCZYK T, KULIG A et al. An association between the matrix metalloproteinase 1 promoter gene polymorphism and lymphnode metastasis in breast cancer. J Exp Clin Cancer Res 2004; 23: 121-125.

[34] PRZYBYLOWSKA K, KLUCZNA A, ZADROZNY M, KRAWCZYK T, KULIG A et al. Polymorphisms of the promoter regions of matrix metalloproteinases genes MMP-1 and MMP-9 in breast cancer. Breast Cancer Res Treat 2006; 95: 65-72. http://dx.doi.org/10.1007/s10549-005-9042-6

[35] HUGHES S, AGBAJE O, BOWEN RL, HOLLIDAY DL, SHAW JA et al. Matrix metalloproteinase single-nucleotide polymorphisms and haplotypes predict breast cancer progression. Clin Cancer Res 2007; 13: 6673-6680. http://dx.doi. org/10.1158/1078-0432.CCR-07-0884

[36] PRICE SJ, GREAVES DR, WATKINS H Identification of novel, functional genetic variants in the human matrix metalloproteinase-2 gene - Role of Sp1 in allele-specific transcriptional regulation. J Biol Chem 2001; 276: 7549-7558. http://dx.doi. org/10.1074/jbc.M010242200

[37] GRIEU F, LI WQ, IACOPETTA B Genetic polymorphisms in the MMP-2 and MMP-9 genes and breast cancer phenotype. Breast Cancer Res Treat 2004; 88: 197-204. http://dx.doi. org/10.1007/s10549-004-0595-6

[38] ZHOU YF, YU CY, MIAO XP, TAN W, LIANG G et al. Substantial reduction in risk of breast cancer associated with genetic polymorphisms in the promoters of the matrix metalloproteinase-2 and tissue inhibitor of metalloproteinase-2 genes. Carcinogenesis 2004; 25: 399-404.

[39] BEEGHLY-FADIEL A, LU W, LONG JR, SHU XO, ZHENG Y et al. Matrix Metalloproteinase-2 Polymorphisms and Breast Cancer Susceptibility. Cancer Epidemiol Biomarkers Prev 2009; 18: 1770-1776. http://dx.doi.org/10.1158/1055-9965. EPI-09-0125

[40] DELGADO-ENCISO I, CEPEDA-LOPEZ FR, MONRROYGUIZAR EA, BAUTISTA-LAM JR, ANDRADE-SOTO M et al. Matrix metalloproteinase-2 promoter polymorphism is associated with breast cancer in a Mexican population. Gynecol Obstet Invest 2008; 65: 68-72. http://dx.doi.org/ $10.1159 / 000108282$

[41] ROEHE AV, FRAZZON APG, AGNES G, DAMIN AP, HARTMAN AA et al. Detection of polymorphisms in the promoters of matrix metalloproteinases 2 and 9 genes in breast cancer in South Brazil: preliminary results. Breast Cancer Res Treat 2007; 102: 123-124. http://dx.doi.org/10.1007/s10549-006$\underline{9273-1}$ 
[42] KRIPPL P, LANGSENLEHNER U, RENNER W, YAZDANIBIUKI B, KOPPEL $\mathrm{H}$ et al. The $5 \mathrm{~A} / 6 \mathrm{~A}$ polymorphism of the matrix metalloproteinase 3 gene promoter and breast cancer. Clin Cancer Res 2004; 10: 3518-3520. http://dx.doi. org/10.1158/1078-0432.CCR-04-0010

[43] LEI HX, ZALOUDIK J, VORECHOVSKY I Lack of association of the-1171 (5A) allele of the MMP3 promoter with breast cancer. Clin Chem 2002; 48: 798-799.

[44] BEEGHLY-FADIEL A, SHU XO, LONG JR, LI C, CAI QY et al. Genetic polymorphisms in the MMP-7 gene and breast cancer survival. Int J Cancer 2009; 124: 208-2014. http://dx.doi. org/10.1002/ijc.23859

[45] ZHANG JH, JIN X, FANG SM, WANG R, LI Y et al. The functional polymorphism in the matrix metalloproteinase-7 promoter increases susceptibility to esophageal squamous cell carcinoma, gastric cardiac adenocarcinoma and non-small cell lung carcinoma. Carcinogenesis 2005; 26: 1748-1753. http://dx.doi.org/10.1093/carcin/bgi144

[46] BEEGHLY-FADIEL A, LONG JR, GAO YT, LI C, QU SM et al. Common MMP-7 polymorphisms and breast cancer susceptibility: A multistage study of association and functionality. Cancer Res 2008; 68: 6453-6459. http://dx.doi. org/10.1158/0008-5472.CAN-08-0636

[47] BALBIN M, FUEYO A, TESTER AM, PENDAS AM, PITIOT AS et al. Loss of collagenase-2 confers increased skin tumor susceptibility to male mice. Nat Genet 2003; 35: 252-257. http://dx.doi.org/10.1038/ng1249

[48] MONTEL V, KLEEMAN J, AGARWAL D, SPINELLA D, KAWAI $\mathrm{K}$ et al. Altered metastatic behavior of human breast cancer cells after experimental manipulation of matrix metalloproteinase 8 gene expression. Cancer Res 2004; 64: 1687-1694. http://dx.doi.org/10.1158/0008-5472.CAN-03$\underline{2047}$

[49] WANG H, PARRY S, MACONES G, SAMMEL MD, FERRAND PE et al. Functionally significant SNP MMP8 promoter haplotypes and preterm premature rupture of membranes (PPROM). Hum Mol Genet 2004; 13: 2659-2669. http://dx.doi. org/10.1093/hmg/ddh287

[50] DECOCK J, LONG J-R, LAXTON RC, SHU X-O, HODG$\mathrm{KINSON} \mathrm{C}$ et al. Association of matrix metalloproteinase- 8 gene variation with breast cancer prognosis. Cancer Res 2007; 67: 10214-21. http://dx.doi.org/10.1158/0008-5472.CAN-07$\underline{1683}$

[51] ZHANG BP, YE S, HERRMANN SM, ERIKSSON P, DE MAAT $M$ et al. Functional polymorphism in the regulatory region of gelatinase $\mathrm{B}$ gene in relation to severity of coronary atherosclerosis. Circulation 1999; 99: 1788-1794.

[52] BEEGHLY-FADIEL A, LU W, SHU XO, LONG JR, CAI QY et al. MMP9 polymorphisms and breast cancer risk: a report from the Shanghai Breast Cancer Genetics Study.
Breast Cancer Res Treat 2011; 126: 507-513. http://dx.doi. org/10.1007/s10549-010-1119-1

[53] JORMSJO S, YE S, MORITZ J, WALTER DH, DIMMELER $S$ et al. Allele-specific regulation of matrix metalloproteinase12 gene activity is associated with coronary artery luminal dimensions in diabetic patients with manifest coronary artery disease. Circ Res 2000; 86: 998-1003.

[54] SHIN AS, CAI QY, SHU XO, GAO YT, ZHENG W Genetic polymorphisms in the matrix metalloproteinase 12 gene (MMP12) and breast cancer risk and survival: the Shanghai Breast Cancer Study. Breast Cancer Res 2005; 7: R506-R512. http://dx.doi.org/10.1186/bcr1033

[55] MARCHENKO GN, MARCHENKO ND, STRONGIN AY The structure and regulation of the human and mouse matrix metalloproteinase-21 gene and protein. Biochem J 2003; 372: 503-515. http://dx.doi.org/10.1042/BJ20030174

[56] SHAGISULTANOVA EI, NOVIKOVA IA, SIDORENKO YS, MARCHENKO GN, STRONGIN AY et al. The matrix metalloproteinase-21 gene $572 \mathrm{C} / \mathrm{T}$ polymorphism and the risk of breast cancer. Anticancer Res 2004; 24: 199-201.

[57] PENG B, CAO LH, MA XP, WANG WZ, WANG D et al. Metaanalysis of association between matrix metalloproteinases 2, 7 and 9 promoter polymorphisms and cancer risk. Mutagenesis 2010; 25: 371-379. http://dx.doi.org/10.1093/mutage/geq015

[58] PENG B, CAO LH, WANG WZ, XIAN LL, JIANG DK et al. Polymorphisms in the promoter regions of matrix metalloproteinases 1 and 3 and cancer risk: a meta-analysis of 50 case-control studies. Mutagenesis 2010; 25: 41-48. http:// dx.doi.org/10.1093/mutage/gep041

[59] MCCOLGAN P, SHARMA P Polymorphisms of matrix metalloproteinases 1, 2, 3 and 9 and susceptibility to lung, breast and colorectal cancer in over 30,000 subjects. Int J Cancer 2009; 125: 1473-1478. http://dx.doi.org/10.1002/ijc.24441

[60] ZHOU P, DU LF, LV GQ, YU XM, GU YL et al. Current evidence on the relationship between four polymorphisms in the matrix metalloproteinases (MMP) gene and breast cancer risk: a meta-analysis. Breast Cancer Res Treat 2011; 127: 813-818. http://dx.doi.org/10.1007/s10549-010-1294-0

[61] HE T No evidence indicated that polymorphisms of the matrix metalloproteinases genes were associated with breast cancer risk: an update. Breast Cancer Res Treat 2011, 128: 589-59. http://dx.doi.org/10.1007/s10549-011-1406-5

[62] WU XY, CHEN MB, WANG LQ, XU Y, TAN Y et al. Polymorphisms of matrix metalloproteinases (MMP) gene and breast cancer risk: appraisal of a recent meta-analysis. Breast Cancer Res Treat 2011, 130: 357-358. http://dx.doi.org/10.1007/ $\underline{\text { 10549-011-1617-9 }}$

[63] MAVADDATN,ANTONIOUAC,EASTONDF, GARCIA-CLOSAS M. Genetic susceptibility to breast cancer. Mol Oncol 2010, 4: 174-191. http://dx.doi.org/10.1016/j.molonc.2010.04.011 